\title{
Splenectomy improves liver fibrosis via tumor necrosis factor superfamily 14 (LIGHT) through the JNK/TGF- $\beta 1$ signaling pathway
}

\author{
Qing-shan Liang ${ }^{1}$, Jian-Gang Xie ${ }^{2}$, ChaoPing Yu², ZhuSheng Feng ${ }^{2}$, JingChang $\mathrm{Ma}^{3}$, Yuan Zhang ${ }^{4,5}$, Dong Wang ${ }^{1}$,
} JianGuo Lu', Ran Zhuang ${ }^{3,4}$ and Jikai Yin (1)

\begin{abstract}
Splenectomy has been reported to improve liver fibrosis in patients with cirrhosis and hypersplenism. However, the mechanisms remain unclear. Tumor necrosis factor superfamily 14 (TNFSF14; also known as LIGHT) is highly expressed in the context of fibrosis and promotes disease progression in patients with fibrotic diseases such as pulmonary and skin fibrosis. Here, we determined whether splenectomy controls the production of LIGHT to improve liver fibrosis. Splenectomy reduced serum LIGHT levels in cirrhotic patients with hypersplenism and a ConA-induced liver fibrosis mouse model. Blocking LIGHT resulted in the downregulation of TGF- $\beta 1$ in RAW264.7 cells. LIGHT treatment of RAW264.7 and JS1 cells in coculture regulated transforming growth factor- $\beta 1$ (TGF- $\beta 1$ ) expression through the activation of JNK signaling. Small interfering RNA-mediated silencing of lymphotoxin $\beta$ receptor (LTBR) in macrophages resulted in pronounced decreases in the levels of fibrosis and ASMA in JS1 cells. These results indicated that LIGHT bound to LT $\beta R$ and drove liver fibrosis in vitro. Blocking TGF- $\beta 1$ abolished the effect of LIGHT in vitro. Furthermore, the administration of recombinant murine LIGHT protein-induced liver fibrosis with splenectomy, while blocking LIGHT without splenectomy improved liver fibrosis in vivo, revealing that the decrease in fibrosis following splenectomy was directly related to reduced levels of LIGHT. Thus, high levels of LIGHT derived from the spleen and hepatic macrophages activate JNK signaling and lead to increased TGF- $\beta 1$ production in hepatic macrophages.

Splenectomy attenuates liver fibrosis by decreasing the expression of LIGHT.
\end{abstract}

\section{Introduction}

Clinical complications such as splenomegaly and hypersplenism often occur in patients with cirrhosis $^{1,2}$. The observed association between the liver and spleen in terms of anatomy, histology, and immunity has been defined as the liver-spleen axis ${ }^{3}$. Previous studies have suggested that abnormal splenic functions occur through a variety of immunological mechanisms to promote liver

\footnotetext{
Correspondence: JianGuo Lu (lujguo@fmmu.edu.cn) or

Ran Zhuang (fmmuzhr@fmmu.edu.cn) or Jikai Yin (Tdyjk07@fmmu.edu.cn) ${ }^{1}$ Department of General Surgery, The Second Affiliated Hospital of Air Force Military Medical University, 710038 Xi'an, Shaanxi, China

${ }^{2}$ Department of Emergency, The First Affiliated Hospital of Air Force Military Medical University, 710032 Xi'an, Shaanxi, China

Full list of author information is available at the end of the article

These authors contributed equally: Qing-shan Liang, Jian-Gang Xie
}

fibrosis and the development of cirrhosis ${ }^{4,5}$. Aoyama et al. $^{6}$ reported that spleen-derived lipocalin-2 has an important role in regulating immune tolerance in the liver during liver fibrosis development. Furthermore, both clinical and animal studies indicate splenic involvement in hepatic fibrosis mainly through the production and secretion of TGF- $\beta 1$ by splenic macrophages ${ }^{7,8}$.

Splenectomy has been used to improve and treat fatal complications associated with portal hypertension in cirrhosis patients, such as bleeding and esophageal and gastric varices ${ }^{3,9}$. Additionally, there have been many reports showing that splenectomy may be an efficient method to improve liver functions and the prognosis of esophageal varices ${ }^{10,11}$. Recently, an animal study showed that splenectomy improved hepatic fibrosis by affecting

\section{(c) The Author(s) 2021}

\footnotetext{
(c) Open Access This article is licensed under a Creative Commons Attribution 4.0 International License, which permits use, sharing, adaptation, distribution and reproduction cc) in any medium or format, as long as you give appropriate credit to the original author(s) and the source, provide a link to the Creative Commons license, and indicate if changes were made. The images or other third party material in this article are included in the article's Creative Commons license, unless indicated otherwise in a credit line to the material. If material is not included in the article's Creative Commons license and your intended use is not permitted by statutory regulation or exceeds the permitted use, you will need to obtain permission directly from the copyright holder. To view a copy of this license, visit http://creativecommons.org/licenses/by/4.0/.
} 
the cytokines secreted by macrophages ${ }^{12}$. Nomura et al. ${ }^{8}$ reported that splenectomy reversed liver fibrosis and improved the systemic immune status in cirrhotic patients. However, the mechanism by which splenectomy improves hepatic fibrosis remains unclear.

As a member of the TNF receptor superfamily, LIGHT was first described as a protein secreted by activated $\mathrm{T}$ lymphocytes that was cleaved by matrix metalloprotein- 9 $\left(\right.$ MMP-9) ${ }^{13}$. LIGHT exerts biological effects by binding to two receptors in the TNF receptor superfamily, herpesvirus entry mediator (HVEM, or TNFRSF14) and lymphotoxin $\beta$ receptor (LT $\beta R$, or TNFRSF3), which are broadly expressed in macrophages, fibroblastic reticular cells, dendritic cells, neutrophils, and $\mathrm{T}$ and $\mathrm{B}$ cells ${ }^{14,15}$. LIGHT may drive fibrosis by increasing the number of macrophages at inflamed sites and increase macrophage expression of TGF- $\beta$, MMP-9, and IL- $8{ }^{16,17}$. Previous studies have shown that LIGHT promotes fibrosis in lung and skin tissues. Blocking the binding of LIGHT to either of its receptors improves the clinical symptoms of fibrosis in the lung and skin ${ }^{18,19}$. Additionally, Herro et al. ${ }^{15}$ found the role of LIGHT in maintaining or perpetuating fibrosis and considered LIGHT to be an attractive target for fibrosis therapies in patients with cirrhosis. However, the relationship between splenectomy and LIGHT in the improvement of liver fibrosis and the underlying mechanisms remain largely unclear.

In this study, we investigated the effects of splenectomy in both cirrhotic patients and a mouse model of ConAinduced liver fibrosis to determine whether LIGHT, JNK, and TGF- $\beta 1$ were necessary for the protective effects of splenectomy against liver fibrosis. Additionally, the direct role of LIGHT in liver fibrosis was investigated by the administration of murine LIGHT protein in vivo.

\section{Materials and methods \\ Patients and clinical specimens}

Normal sera were obtained from 23 healthy volunteers. Serum was collected from 23 cirrhotic patients with hypersplenism $1 \mathrm{~h}$ before and $10 \mathrm{~d}$ after splenectomy at the Department of General Surgery in the Second Affiliated Hospital of Air Force Military Medical University (Xi'an, China). To ensure adequate power to detect a prespecified effect, the sample size was chosen using the Power and Sample Size Program. The levels of LIGHT, TGF- $\beta 1$, IL- 6 , and TNF- $\alpha$ were measured by enzymelinked immunosorbent assay (ELISA). Patients with primary liver cancer, congenital heart disease, diabetes, chronic respiratory diseases, alcohol abuse, or a history of drug use were excluded. There was no history of hepatocirrhosis or splenomegaly in any volunteer, and liver biochemical analyses were normal. All subjects provided informed consent, and the experimental procedures were approved by the Research Ethics Committee of the
Second Affiliated Hospital of Air Force Military Medical University. Patient information is presented in Supplemental Material S1.

\section{Animal models of liver fibrosis with splenectomy}

Six- to eight-week-old female C57BL/6 mice were purchased from the Laboratory Animal Center of Air Force Military Medical University. The mice were kept in a specific pathogen-free facility at $21 \pm 2{ }^{\circ} \mathrm{C}$ with a 12 -h dark/light cycle. Concanavalin A (ConA) was administered to the mice via the tail vein at a dose of $12.5 \mathrm{mg} / \mathrm{kg}$ weekly for 7 consecutive weeks. A total of 24 mice were randomly assigned to four groups: Blank, ConA model, ConA combined with sham, and ConA combined with splenectomy. Each group included six mice. The sham group was only subjected to an open-and-close abdominal operation. Splenectomy was conducted 1 day after the fifth ConA injection. To mimic immune damage in vivo, ConA injections were continued until the mice were sacrificed. All mice were sacrificed on the first day of the 8th week, and blood and liver tissue samples were collected. Alanine aminotransferase (ALT), aspartate aminotransferase (AST), and platelet (PLT) levels were analyzed by Servicebio Co. (Wuhan, China). Sera were collected from mice at various times during the course of the ConA injections $(0,2,4,5$, and 7 weeks) and after splenectomy to measure the level of LIGHT. Liver tissues were subjected to immunofluorescence staining for F4/80 and TGF- $\beta 1$, immunohistochemical staining for $\alpha$ smooth muscle actin ( $\alpha \mathrm{SMA})$, and Sirius red staining. The experimental procedures and animal care were carried out strictly in accordance with the related ethical regulations of Air Force Military Medical University. There were no animals excluded from analyses, and no blinding was carried out, but the data analysts were blinded to the groupings.

\section{Splenectomy}

All mice were subjected to isoflurane anesthesia before splenectomy. Splenectomy was performed as described previously ${ }^{20}$. All mice were euthanized on the first day of the 8th week, and surgical procedures were conducted under completely sterile conditions.

\section{Cell lines and transwell coculture assays}

The murine hepatic macrophage cell line RAW264.7 and murine hepatic stellate cell line JS1 were obtained from the Department of Immunology of Air Force Military Medical University. All cells were cultured in DMEM with $10 \%$ FBS. The cell lines were verified to be authentic.

Recombinant murine LIGHT protein (rLIGHT) was used to stimulate RAW264.7 cells for $0,6,12$, and $24 \mathrm{~h}$ to determine the optimal time for TGF- $\beta 1$ production. TGF$\beta 1$ mRNA was measured by qPCR. The expression of 
JNK and P-JNK in RAW264.7 cells was measured by western blotting.

In addition, LTßR-Ig (Fc-tagged recombinant mouse LT $\beta R$ protein, LT $\beta R$-Ig) was used to block LIGHT in the coculture assay. Splenocytes from C57BL/6 mice were seeded in the upper chambers $\left(5 \times 10^{5}\right)$ of a six-well Transwell plate, while $1 \times 10^{6}$ RAW264.7 cells were seeded in the lower chambers. ConA $(1.5 \mu \mathrm{g} / \mathrm{ml}$; Sigma Chemical Co.) was added to activate $\mathrm{T}$ cells, and $10 \mu \mathrm{g} / \mathrm{ml}$ LT $\beta R$-Ig and control IgG were added to the upper chambers and incubated for $12 \mathrm{~h}^{21}$. The expression of TGF- $\beta 1$ in RAW264.7 cells was measured by qPCR and western blotting.

For LIGHT stimulation, rLIGHT-treated RAW264.7 cells $\left(5 \times 10^{5}\right)$ were seeded in the upper chambers of a sixwell Transwell plate (Corning, Costar 3422), while $1 \times 10^{6}$ JS1 cells were plated in the lower chambers. SP600125 (P-JNK inhibitor) was added to RAW264.7 cells $15 \mathrm{~min}$ before coculture to assess the regulatory effect of JNK signaling on TGF- $\beta 1$ and $\alpha$ SMA expression. Cells were incubated for $12 \mathrm{~h}$ to examine whether TGF- $\beta 1$ produced by rLIGHT-stimulated RAW264.7 cells affected the functions of JS1 cells. Additionally, the levels of TGF- $\beta 1$ were measured in the supernatant of JS1 cells from the coculture assay. rLIGHT (Cat. No. 1794-LT/CF, R\&D Systems) was used at a concentration of $100 \mathrm{ng} / \mathrm{ml}$, and SP600125 (Cat. No.: HY-12041, MedChemExpress) was used at a concentration of $10 \mu \mathrm{M}$.

\section{In vitro knockdown of LT $\beta R$ and HVEM}

For the in vitro knockdown of LT $\beta R$ and HVEM in RAW264.7 cells, siRNAs targeting murine LT $\beta$ R and HVEM and control nonsense siRNA were transfected into RAW264.7 cells using Lipofectamine 3000 (Thermo Scientific, Waltham, MA). After LT $\beta R$ and HVEM knockdown in RAW264.7 cells, the Transwell coculture assay was performed as described above. The level of TGF- $\beta 1$ in RAW264.7 cells was measured by western blotting and immunofluorescence staining. The level of TGF- $\beta 1$ in the culture medium of JS1 cells was measured by ELISA (Baizhoubio, Qingdao, China). $\alpha$ SMA expression in JS1 cells was measured by western blotting. All siRNAs were purchased from Guangzhou RiboBio Co., Ltd. (Guangzhou, China). siRNA vectors were added to the culture medium at a concentration of $100 \mathrm{nM}$. The siRNA targeting sequences were as follows: control nonsense siRNA: CCCTCGAATGTGAATGGAA; siHVEM: GCAA ATGGCCTGAGCAAGT; and siLTßR: GGACACTTCC AGAACACTT.

\section{In vitro TGF- $\beta 1$ inhibition}

The coculture assay included three groups: the control group, which contained RAW264.7 and JS1 cells; the rLIGHT group, in which RAW264.7 and JS1 cells were stimulated by rLIGHT; and the TGF- $\beta 1$-NAB (anti-TGF$\beta 1$-neutralizing antibody) group, in which RAW264.7 and JS1 cells were treated with rLIGHT and the anti-TGF- $\beta 1$ neutralizing antibody. RAW264.7 cells were pretreated with rLIGHT and/or anti-TGF- 31 -neutralizing antibody for $12 \mathrm{~h}$ and then cocultured with JS1 cells in the Transwell system. The levels of $\alpha$ SMA in JS1 cells were measured by western blotting and immunofluorescence staining. The concentration of both the anti-TGF- $\beta 1$ neutralizing antibody (Cat. No.: MAB1835-SP, R\&D Systems) and rLIGHT protein (Cat. No.: 1794-LT/CF, R\&D Systems) was $100 \mathrm{ng} / \mathrm{ml}$.

\section{Effects of recombinant murine LIGHT on liver fibrosis in vivo}

A total of 24 C57BL/6 mice were randomly assigned to four groups. Except for mice in the control group, all mice received a weekly dose of $12.5 \mathrm{mg} / \mathrm{kg}$ body weight ConA via tail vein injection. Splenectomy was conducted 1 day after the fifth ConA injection. Beginning in the 6th week, the rLIGHT group received a weekly dose of $10 \mu \mathrm{g}$ of rLIGHT, while the ConA group received ConA. In addition, LIGHT was blocked by the administration (i.p.) of $100 \mu \mathrm{g}$ of recombinant LT $\beta \mathrm{R}$ weekly for 7 consecutive weeks ${ }^{16}$.

The mice were sacrificed on the first day of the 8th week, and liver tissue samples were subjected to immunofluorescence staining for $\mathrm{F} 4 / 80+$ and TGF- $\beta 1+$ macrophages, immunohistochemical staining for $\alpha$ SMA, and Sirius red staining. Western blotting was used to measure the expression of TGF- $\beta 1$ and $\alpha$ SMA in liver tissue samples.

\section{Histological staining}

Sirius red staining, immunohistochemical staining, and immunofluorescence staining were performed as described previously ${ }^{22,23}$. Sirius red staining solution was purchased from Servicebio (Wuhan, China). Antibodies against murine F4/80 and TGF- $\beta 1$ were used to immunofluorescently label macrophages and TGF- $\beta 1+$ macrophages in the liver tissue of mice. Antibodies against murine TGF- $\beta 1$ and $\alpha$ SMA were used to immunofluorescently label TGF- $\beta 1$ in RAW264.7 cells and $\alpha$ SMA in JS1 cells, respectively. The following primary antibodies were used: F4/80 (gb11027; Servicebio, Wuhan, China), TGF- $\beta 1$ (ab106582, Abcam, Cambridge, UK), and $\alpha$ SMA (sc-53142; Santa Cruz Biotechnology).

\section{Quantitative reverse transcription PCR (qPCR)}

qPCR analysis was performed as described previously ${ }^{23}$. The data were analyzed by SDS 2.1 software. For all target genes, the relative abundance was determined by the comparative cycle threshold $\mathrm{Ct}$ method and normalized to GAPDH mRNA levels. The primers are presented in Supplemental Material S2. 


\section{Western blot analysis}

Whole-cell lysates of liver tissue or cells were obtained using RIPA lysis buffer containing protease and phosphatase inhibitors. Protein samples were separated by $5 \%$ or $10 \%$ SDS-PAGE. Then, the proteins were transferred onto a polyvinylidene fluoride membrane and incubated with primary antibodies at $4{ }^{\circ} \mathrm{C}$ overnight. Corresponding secondary antibodies were applied to the membrane and incubated for $1 \mathrm{~h}$. Protein bands were visualized by chemiluminescence. The following primary antibodies were used: JNK (sc-571; Santa Cruz Biotechnology), P-JNK (sc6254; Santa Cruz Biotechnology), TGF- $\beta 1$ (ab106582, Abcam), $\alpha$ SMA (sc-53142; Santa Cruz Biotechnology), and $\beta$-actin (sc-47778, Santa Cruz Biotechnology).

\section{Statistical analysis}

The data are expressed as the means \pm standard error of the mean (SEM). For comparison of two groups, Student's $t$-test was used. One-way ANOVA followed by the Tukey multiple comparison test was used to compare the differences between three or more groups. A value of $P<$ 0.05 was considered statistically significant. Statistical analyses were performed using GraphPad Prism 5 (GraphPad Software Inc., San Diego, CA).

\section{Results}

Splenectomy reduces the serum level of LIGHT in cirrhotic patients

ELISA was used to analyze the serum levels of LIGHT and other fibrotic markers, including TGF- $\beta 1$, IL- 6 , and TNF- $\alpha$, in healthy volunteers and cirrhotic patients before and after splenectomy. The serum levels of LIGHT, TGF$\beta 1$, IL- 6 , and TNF- $\alpha$ were all significantly increased in presplenectomy patients compared to healthy individuals (Fig. 1a-d), and LIGHT was more significantly increased in presplenectomy patients than in the other patients $(P<$ $0.001)$.

However, compared with those of presplenectomy patients, LIGHT and TGF- $\beta 1$ levels were decreased significantly in patients after splenectomy (Fig. 1a, b), while no significant difference in IL- 6 or TNF- $\alpha$ was observed after splenectomy $(P>0.05)$ (Fig. 1c, d).

\section{The ConA-induced liver fibrosis and thrombocytopenia animal model is suitable for analyzing the effect of splenectomy on liver fibrosis and hypersplenism}

After treatment of the mice with ConA for 7 continuous weeks, the liver surface was gray and granular in the ConA and sham groups, whereas mice subjected to splenectomy had smooth hepatic surfaces (Fig. 2a). ALT and AST were significantly increased $(P<0.001)$ following ConA treatment but markedly declined after splenectomy $(P<0.01)$ (Fig. 2b). The platelet count was markedly decreased after ConA treatment $(P<0.01)$ (Fig. 2c), which was concomitant with significantly increased serum ALT and AST levels, resembling hypersplenism with cirrhosis in humans. Most importantly, weekly injection of ConA obviously increased the serum concentration of LIGHT, which then decreased significantly after splenectomy (Fig. 2d). Moreover, immunohistochemical staining of $\alpha$ SMA revealed a significant increase in the number of activated myofibroblasts after ConA treatment $(P<0.001)$, while splenectomy significantly decreased the number of these cells $(P<0.05)$, and the Sirius red staining results were in accordance with the immunohistochemical staining results (Fig. 2e). The protein levels of TGF- $\beta 1$ and $\alpha$ SMA were significantly increased after treatment with ConA, but splenectomy markedly decreased these levels (Fig. 2f). Additionally, immunofluorescence staining revealed increases in the percentages of $\mathrm{F} 4 / 80+$ and $\mathrm{F} 4 / 80$ $+/$ TGF- $\beta 1+$ macrophages after ConA treatment, which were reduced by splenectomy $(P<0.05)$ (Fig. $2 \mathrm{~g})$.

\section{LIGHT induces JNK phosphorylation and increases TGF- $\beta 1$ in macrophages}

To confirm whether LIGHT regulated liver fibrosis, the murine haptic macrophage cell line RAW264.7 was used for in vitro experiments. Because macrophages have a major role in promoting liver fibrosis ${ }^{24}$ and LIGHT was significantly increased in both cirrhotic patients and liver fibrosis animal models, we hypothesized that macrophages may be affected by LIGHT. First, we stimulated RAW264.7 cells with rLIGHT for $0,6,12$, and $24 \mathrm{~h}$ to determine the optimal stimulation time. TGF- $\beta 1$ mRNA levels were significantly upregulated at $12 \mathrm{~h}(P<0.01)$ (Fig. 3a). Because TGF- $\beta 1$ production correlates with P-JNK activity ${ }^{25}$, we postulated that TGF- $\beta 1$ expression may be regulated by alterations in JNK signaling after rLIGHT treatment. Surprisingly, the level of phosphorylated JNK (P-JNK) was also significantly upregulated in the cells $(P<$ 0.01) (Fig. 3b). Conversely, blocking LIGHT with LT $\beta$ R-Ig in splenocyte and RAW264.7 cell cocultures resulted in decreased levels of TGF- $\beta 1$ in RAW264.7 cells (Fig. 3c, d).

\section{LIGHT upregulates TGF- $\beta 1$ in vitro to promote liver fibrosis through the JNK/P-JNK signaling pathway}

To explore the potential chemotactic effect of LIGHT in liver fibrosis, we used a Transwell coculture assay with RAW264.7 cells in the upper chamber and JS1 cells in the lower chamber. The expression of TGF- $\beta 1$ and P-JNK in RAW264.7 cells was upregulated by LIGHT stimulation $(P<0.001)$ (Fig. 4a). Because TGF- $\beta 1$ passed through the pores of the Transwell inserts from the upper to lower chambers, we hypothesized that the level of TGF- $\beta 1$ was increased in JS1 cell culture medium. ELISA analysis showed that TGF- $\beta 1$ was significantly increased in JS1 cell culture medium $(P<0.01)$ (Fig. 4b). Additionally, western blotting showed that the expression of $\alpha \mathrm{SMA}$ was 


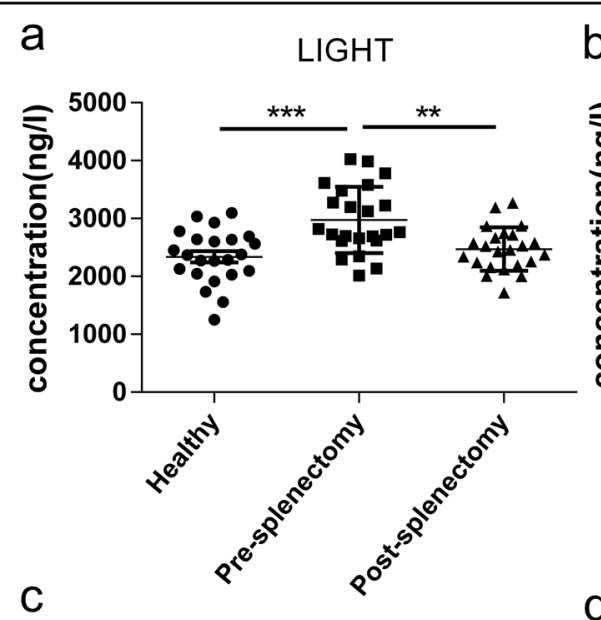

C

IL-6

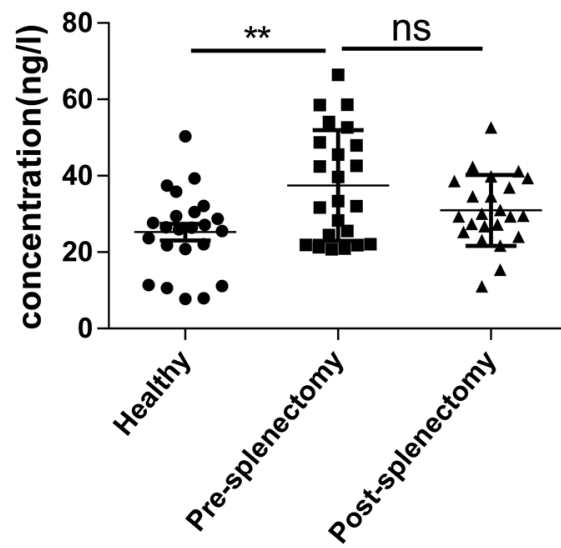

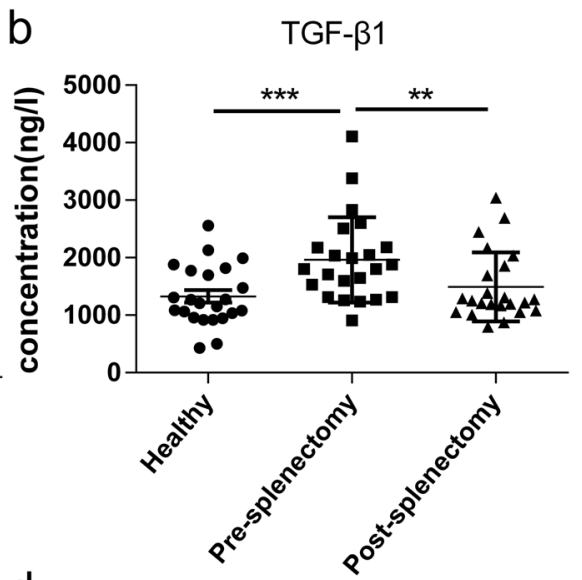

d

TNF- $\alpha$

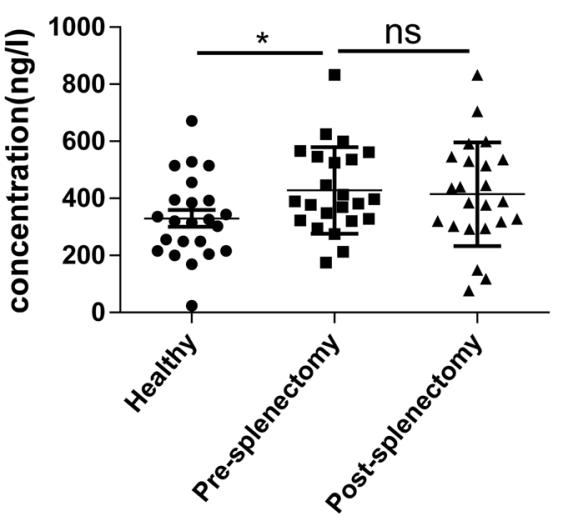

Fig. 1 Effect of splenectomy on the profibrotic factors LIGHT, TGF- $\beta \mathbf{1} 1$, IL-6, and TNF-a. a-d Serum concentrations of LIGHT, TGF- $\beta 1$, IL-6, and TNF-a in healthy controls. Healthy (healthy volunteers), presplenectomy patients (cirrhotic patients before splenectomy), and postsplenectomy patients (cirrhotic patients after splenectomy). Cohort 3: $n=23$, means \pm SEM, ${ }^{*} P<0.05,{ }^{* *} P<0.01,{ }^{* * *} P<0.001$.

significantly upregulated in JS1 cells $(P<0.05)$ (Fig. 4c). Immunofluorescence analysis also showed a significant increase in the number of $\alpha$ SMA-positive cells among JS1 cells $(P<0.01)$ (Fig. 4 d). In contrast, the addition of the JNK inhibitor SP600125 to RAW264.7 cells reversed these effects.

\section{LIGHT uses LT $\beta R$, but not HVEM to drive liver fibrosis}

LIGHT has two receptors in the TNFR superfamily (LTRR/TNFRSF3 and HVEM/TNFRSF14 ${ }^{19}$ ), both of which are expressed by macrophages ${ }^{15}$. To determine whether LIGHT drove liver fibrosis through one or both of its receptors, siRNAs against murine LT $\beta R$ and HVEM and nonsense siRNA were transfected into RAW264.7 cells for $48 \mathrm{~h}$, and then qPCR was used to measure LT $\beta \mathrm{R}$ and HVEM mRNA expression. As a result, the levels of both LT $\beta$ R and HVEM were significantly reduced $(P<$ 0.01) (Fig. 5a, b). These results indicated a successful knockdown of the target genes. Next, rLIGHT was used to stimulate RAW264.7 cells transfected with LT $\beta$ R and
HVEM siRNA. Compared with those in the SiNC group, the levels of TGF- $\beta 1$ and $\alpha$ SMA were significantly upregulated in the SiNC + LIGHT groups $(P<0.01)$. Compared with that in the SiNC + LIGHT group, the expression of TGF- $\beta 1$ and/or $\alpha$ SMA was significantly downregulated in rLIGHT-treated RAW264.7 cells transfected with LT $\beta$ R siRNA, and there was no significant difference in the HVEM siRNA group (Fig. 5c-f). Taken together, these results suggest a mechanism by which LIGHT promotes fibrosis through direct binding to LT $\beta R$ but not HVEM on hepatic macrophages to increase TGF- $\beta 1$ expression.

\section{LIGHT directly induces liver fibrosis via TGF- $\beta 1$}

Murine hepatic macrophages are capable of producing TGF- $\beta 1$, which participates in the development of liver fibrosis $^{26,27}$. Therefore, we determined whether LIGHT induced liver fibrosis in vitro by a TGF- $\beta 1$-dependent mechanism. We used an anti-TGF- $\beta 1$-neutralizing antibody in the RAW264.7/JS1 cell coculture system to 


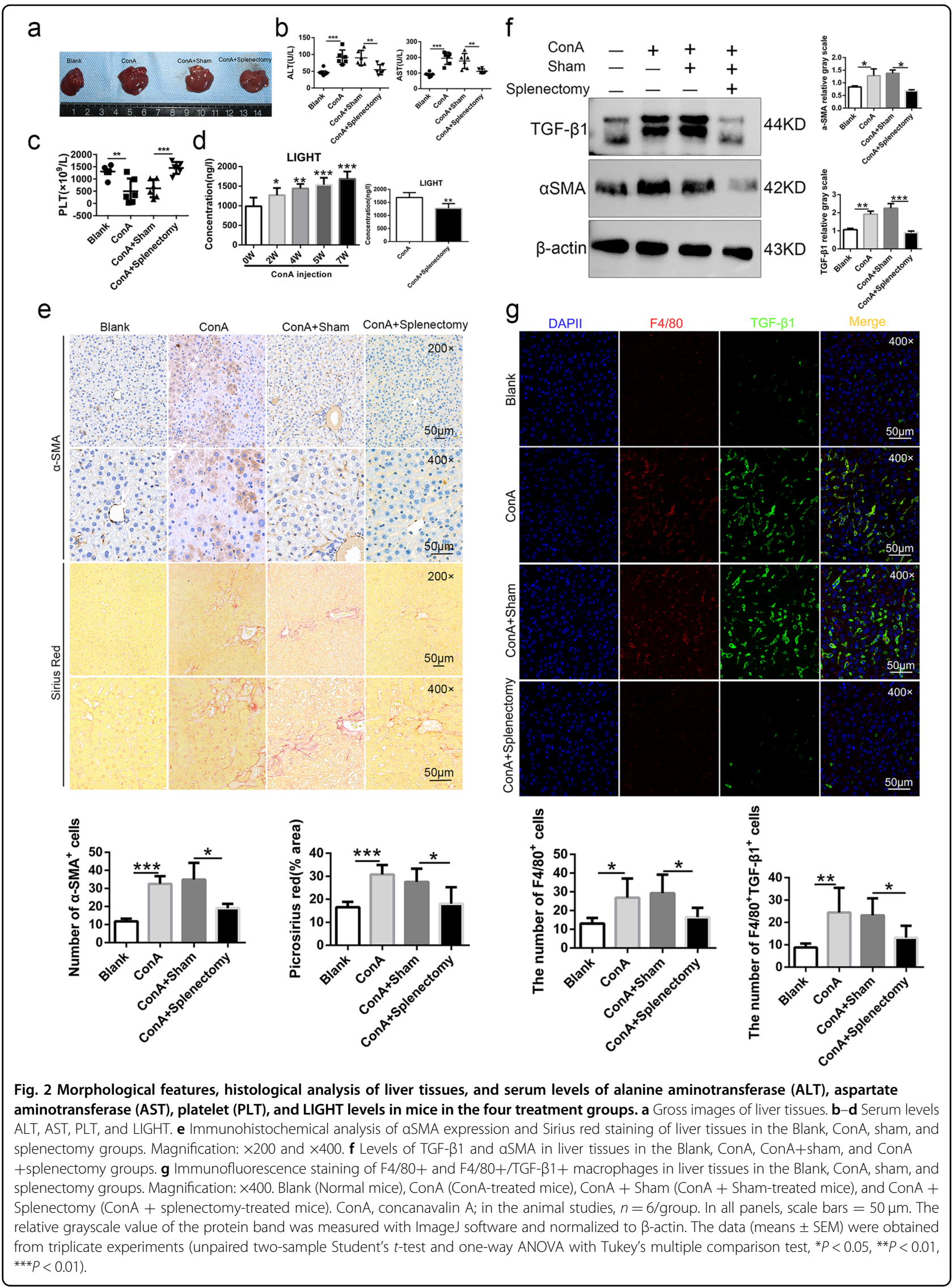




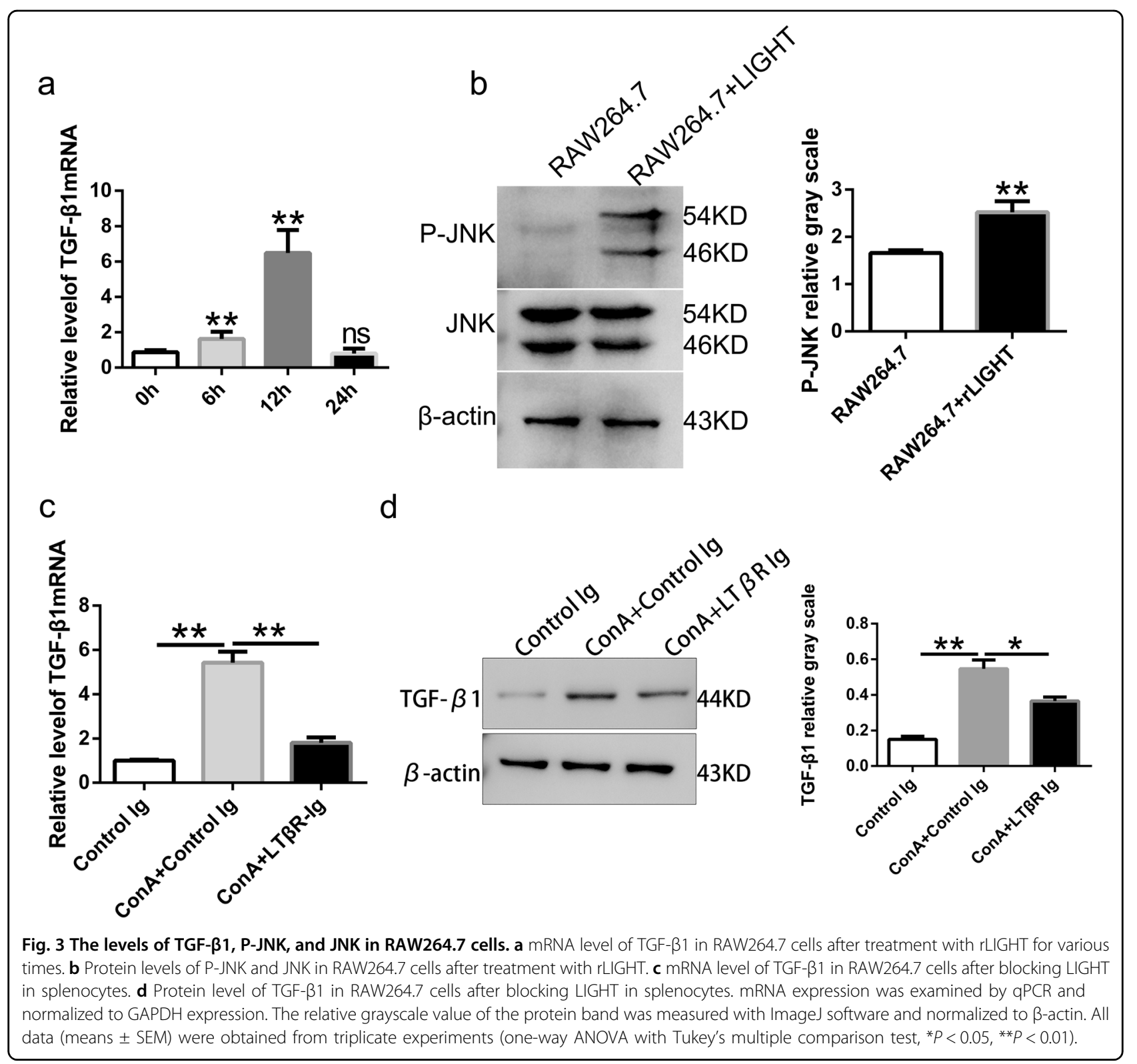

neutralize the effects of TGF- $\beta 1$ after treatment with rLIGHT. Surprisingly, both western blotting and immunofluorescence showed decreased expression of $\alpha S M A$ in JS1 cells (Fig. 6a, b). Thus, LIGHT induced liver fibrosis through TGF- $\beta 1$-dependent signaling.

\section{LIGHT promotes fibrotic factors in vivo}

Splenectomy improved liver fibrosis and induced a significant decline in the serum LIGHT level; thus, we assessed whether LIGHT had direct roles in exacerbating liver fibrosis after splenectomy in vivo. First, rLIGHT was administered through the angular vein to mice with liver fibrosis induced by ConA. Compared with those in the Blank group, hepatic volume was increased, and liver surfaces were gray and granular after ConA and rLIGHT treatment (Fig. 7a). Compared with those in the splenectomy group, F4/80+ and F4/80+/TGF- $\beta 1+$ macrophages were significantly increased after rLIGHT treatment $(P<0.05)($ Fig. $7 \mathrm{~b})$, and worsened liver fibrosis was indicated by Sirius red staining and immunohistochemical staining with a large area of fibrosis after rLIGHT treatment (Fig. 7c). Furthermore, the expression of TGF- $\beta 1$ and $\alpha$ SMA was increased in the rLIGHT group $(P<0.01)$ (Fig. $7 \mathrm{~d})$. Therefore, rLIGHT increased liver fibrosis in mice that underwent splenectomy.

\section{Liver fibrosis is suppressed in LIGHT-deficient animals}

To better understand the effects of LIGHT on liver fibrosis, we used LT $\beta$ R-Ig to block LIGHT in vivo and performed immunohistochemical staining, Sirius red 


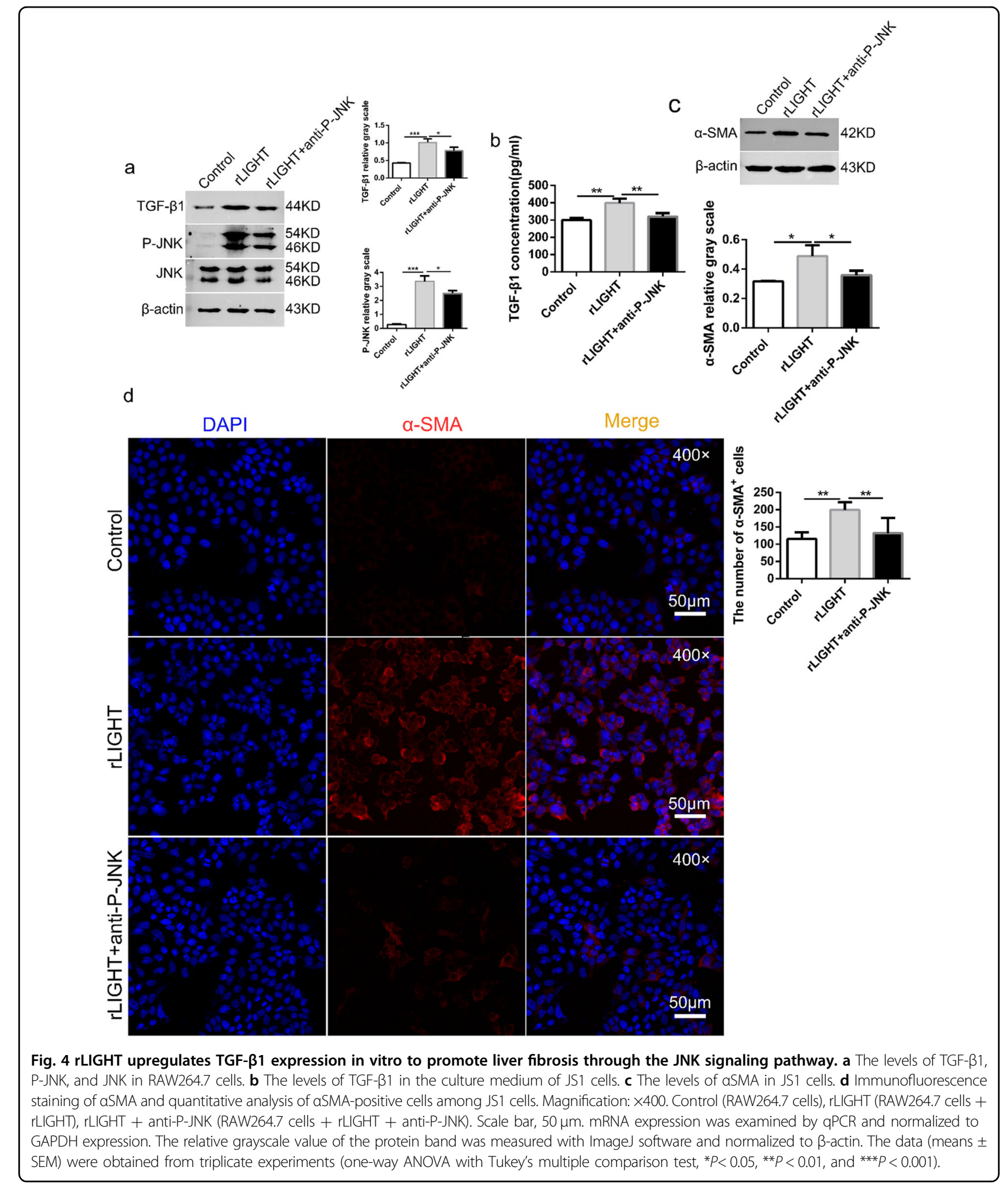

staining, and western blotting to measure the level of $\alpha$ SMA and fibrosis in liver tissue samples. The results showed significantly decreased $\alpha$ SMA levels and fibrosis in the ConA+LT $\beta R$-Ig group (Fig. 8a-c).

\section{Discussion}

Several reports have emphasized the protective effects of splenectomy on liver fibrosis ${ }^{2,8,28-30}$. However, the mechanism remains largely unknown. Here, we examined 


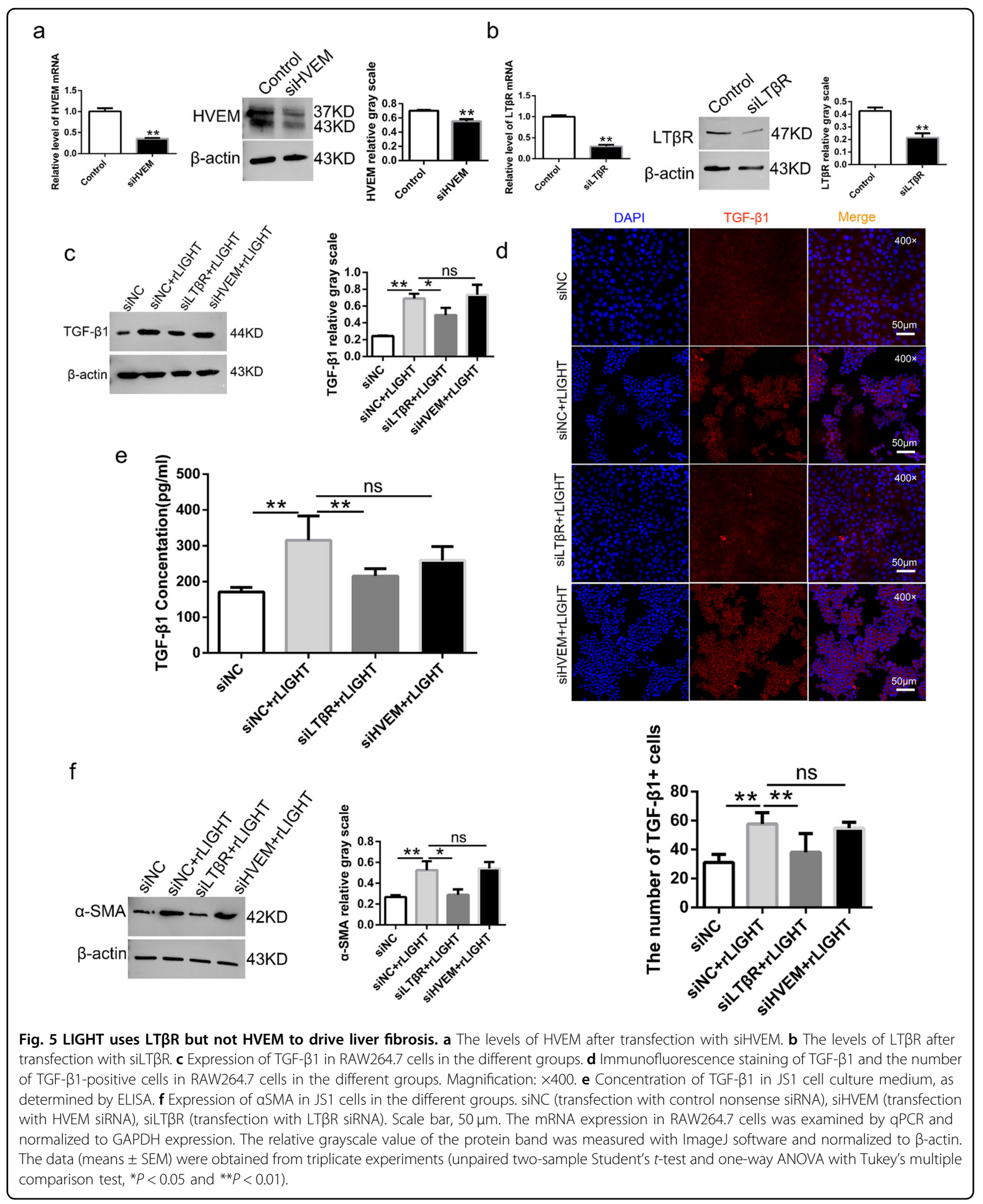

changes in profibrotic cytokines in the serum of cirrhosis patients before and after splenectomy. As profibrotic cytokines, LIGHT ${ }^{15}$, TGF- $\beta 1^{29}$, IL- $6^{31}$, and TNF- $\alpha^{32}$ have major roles in fibrogenesis. We determined that LIGHT, TGF- $\beta 1$, IL- 6 , and TNF- $\alpha$ were all significantly increased in the serum of cirrhotic patients, whereas splenectomy 

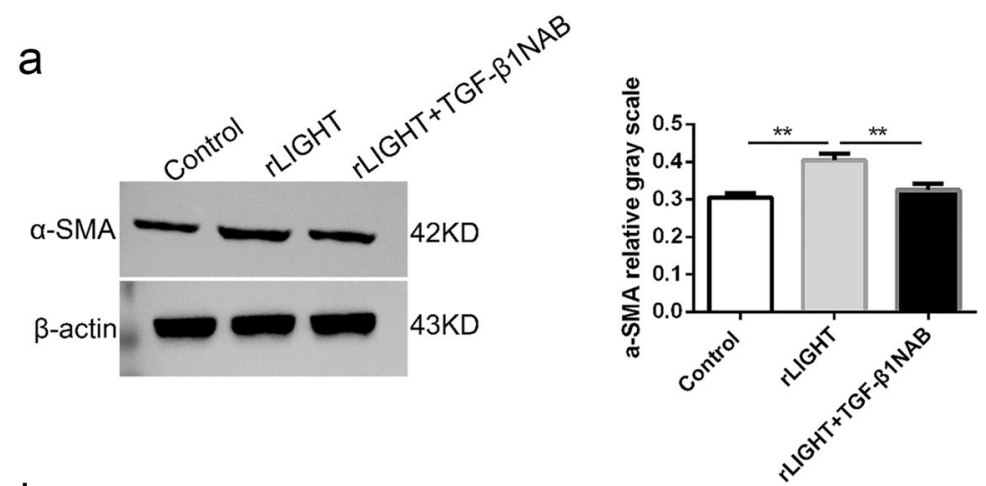

$\mathrm{b}$
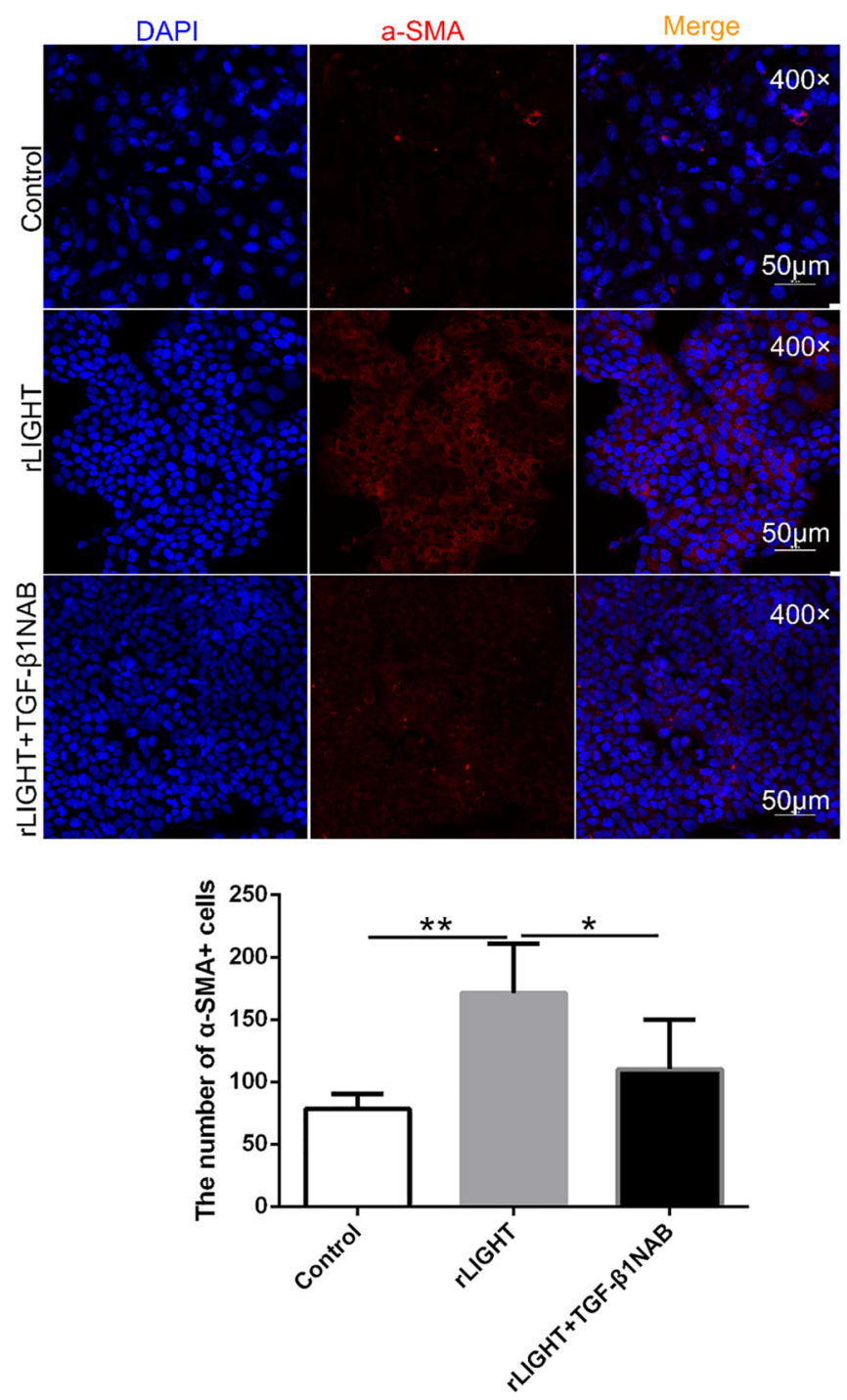

Fig. 6 LIGHT directly induces liver fibrosis via TGF- $\beta$ 1. a The level of ASMA in JS1 cells after treatment with an anti-TGF- $\beta 1$-neutralizing antibody. b Immunofluorescence staining of aSMA in JS1 cells after treatment with an anti-TGF- $\beta 1$-neutralizing antibody. Magnification: $\times 400$. Control (coculture assay of RAW264.7 and JS1 cells); rLIGHT group: (coculture assay of RAW264.7 and JS1 cells with rLIGHT stimulation); rLIGHT+TGF- $\beta 1 N A B$ : (coculture assay of RAW264.7 and JS1 cells treated with rLIGHT and an anti-TGF- $\beta 1$-neutralizing antibody); scale bar, $50 \mu \mathrm{m}$. The relative grayscale value of the protein band was measured with ImageJ software and normalized to $\beta$-actin. The data (means \pm SEM) were obtained from triplicate experiments (one-way ANOVA with Tukey's multiple comparison test, ${ }^{*} P<0.05$ and ${ }^{* *} P<0.01$ ). 


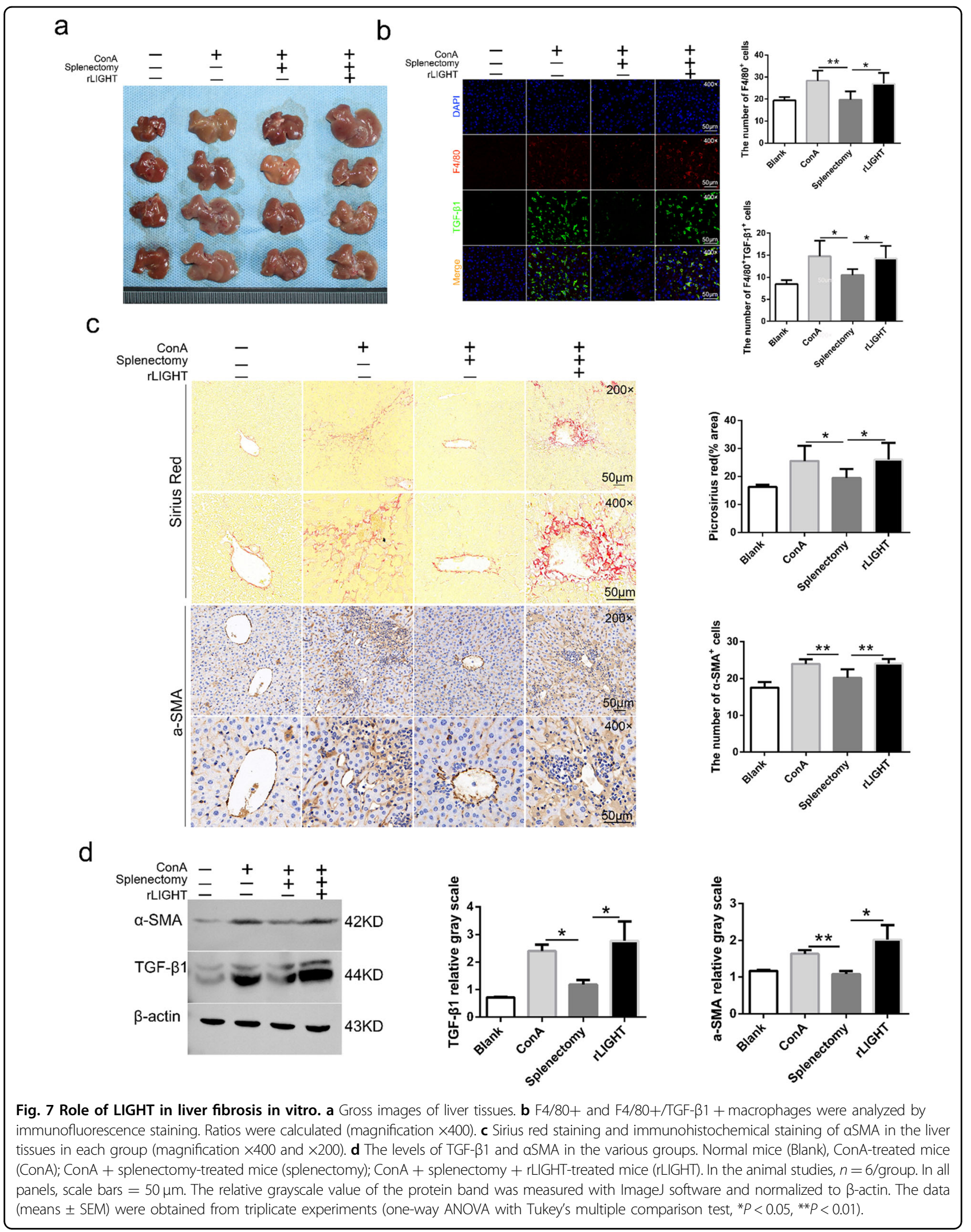



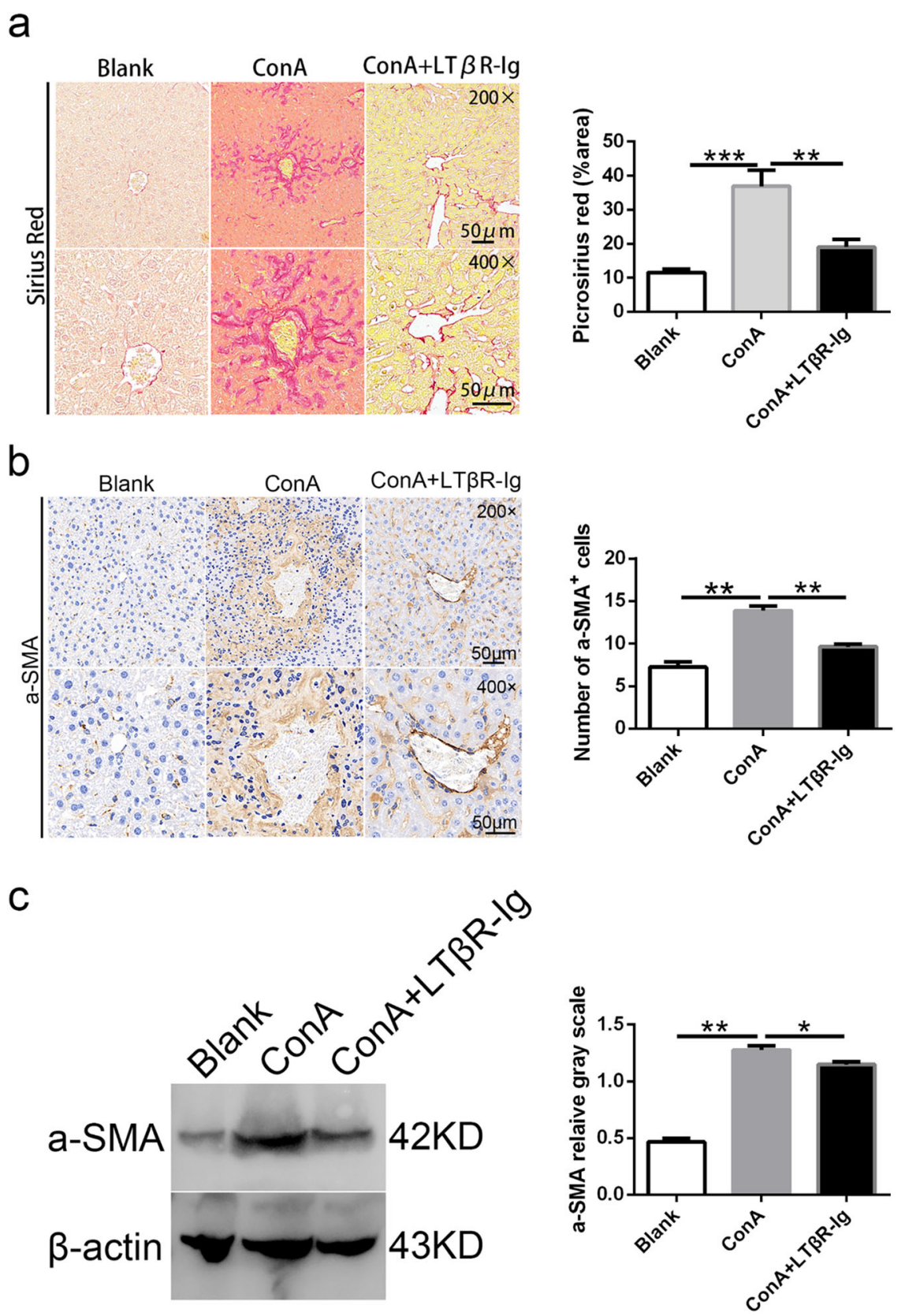

Fig. 8 Histological analysis and the level of a-SMA in liver tissues. a Sirius red staining in liver tissues. $\mathbf{b}$ Immunohistochemical staining of a-SMA in liver tissues. c The levels of a-SMA in liver fibrosis. Normal mice (Blank), ConA-treated mice (ConA), ConA- and LTßR-Ig-treated mice (ConA+LTßRIg). In the animal studies, $n=3 /$ group. In all panels, scale bars $=50 \mu \mathrm{m}$. The relative grayscale value of the protein band was measured with ImageJ software and normalized to $\beta$-actin. The data (means \pm SEM) were obtained from triplicate experiments (one-way ANOVA with Tukey's multiple comparison test, $\left.{ }^{*} P<0.05,{ }^{* *} P<0.01,{ }^{* * *} P<0.001\right)$.

only induced significant decreases in LIGHT and TGF- $\beta 1$. Therefore, splenectomy may be more closely associated with LIGHT and TGF- $\beta 1$. LIGHT is mainly expressed by activated $\mathrm{T}$ cells ${ }^{33}$. However, as the membrane-bound form of LIGHT may be rapidly cleaved in the serum, the cellular and tissue sources of LIGHT can be difficult to identify $^{34}$. Unfortunately, we have no data on the source of LIGHT in serum. Other studies have shown that LIGHT is increased in the serum of patients with various fibrotic and inflammatory diseases ${ }^{34-37}$. Additionally, LIGHT promotes the deposition of collagen in the context of fibrosis by controlling major profibrotic factors such as TGF- $\beta 1$ and is considered to be a potential target to halt the development of fibrosis ${ }^{15,19,34}$. To verify the 
clinical associations among splenectomy, LIGHT, and TGF $\beta$ - 1 , we used a ConA-induced mouse liver fibrosis model. Similar to a previous study of the same model ${ }^{30}$, we also observed significant increases in serum ALT and AST levels and hepatic levels of TGF- $\beta 1$ and $\alpha$ SMA and a significant decrease in PLT in the blood. More importantly, the serum level of LIGHT gradually increased during ConA treatment but decreased following splenectomy. By analyzing cirrhosis patients and an animal model of liver fibrosis, we found that splenectomy induced changes in LIGHT, TGF- $\beta 1$, and liver fibrosis.

Many studies emphasize the relationship between splenectomy, macrophages, and liver fibrosis. For example, Wang et al. ${ }^{30}$ found that splenectomy suppressed the progression of liver fibrosis by affecting the polarization of MDSDs and M2 macrophages. Li et al. ${ }^{12}$ reported that splenectomy improved liver fibrosis by inhibiting the establishment of the M1-dominant phenotype in hepatic macrophages. Yada et $\mathrm{al}^{2}$ found that splenectomy promoted Ly- $6 \mathrm{C}^{\mathrm{lo}}$ macrophage/monocyte accumulation in the fibrotic liver tissue of mice to improve liver fibrosis. Moreover, several effects related to LIGHT and macrophages have been reported in the development of tissue fibrosis. LIGHT induces the accumulation of macrophages and upregulates the level of TGF- $\beta 1$ secreted by macrophages in asthmatic airway remodeling ${ }^{16}$. Other studies have shown that LIGHT acts on macrophages in a membrane-bound form to participate in fibrotic activity ${ }^{17}$. We suspected that splenectomy may also control the expression of LIGHT to affect the secretion of TGF- $\beta 1$ by macrophages in the context of liver fibrosis. We found that rLIGHT affected the hepatic macrophage cell line RAW264.7, promoting the secretion of TGF- $\beta 1$ and upregulating the level of P-JNK in these cells. In the splenocyte and RAW264.7 cell coculture system, blocking LIGHT in splenocytes significantly downregulated the expression of TGF- $\beta 1$. JNK signaling is considered a major modulator of liver fibrosis ${ }^{38}$. Kou et al. ${ }^{39}$ reported that LIGHT inhibited JNK signal activation in beige biogenesis while activating NF- $\mathrm{kB}$ signaling. As an important modulator of the transcription factor AP-1, JNK activation might have a critical role in LIGHT-mediated cellular responses ${ }^{40}$. In our experiment, JNK phosphorylation was increased concomitant with the upregulated expression of TGF- $\beta 1$. The JNK inhibitor strongly reduced TGF- $\beta 1$ expression in RAW264.7 cells and the culture supernatant of JS1 cells. Correspondingly, the level of aSMA in JS1 cells was also decreased. These results showed that the decrease in fibrosis after splenectomy was directly related to reduced levels of LIGHT in vitro. LIGHT promoted the expression of TGF- $\beta 1$ by activating JNK signaling, and the inhibition of JNK abrogated the effects of rLIGHT.

LIGHT has two receptors, HVEM and LT $\beta$, both of which are expressed by macrophages. More importantly, these macrophages often reside close to subepithelial regions where smooth muscle hyperplasia and fibrosis often occur ${ }^{15}$. LIGHT is a major directive protein in the development of pulmonary tissue fibrosis and skin fibrotic activity, and inhibiting the binding of LIGHT to its receptors LT $\beta R$ and HVEM improve fibrosis ${ }^{15,18,19}$. To determine whether LIGHT bound to HVEM and/or LT $\beta R$ in the context of liver fibrosis, we transfected RAW264.7 cells with siRNAs. In a coculture system of HVEM or LT $\beta R$, siRNA-transfected RAW264.7 and JS1 cells treated with rLIGHT, we found that silencing LT $\beta R$ downregulated the expression of TGF- $\beta 1$ in RAW264.7 cells and $\alpha$ SMA in JS1 cells, while no significant difference was observed by silencing HVEM. Therefore, LIGHT bound to LT $\beta R$ but not HVEM in RAW264.7 cells to induce the expression of TGF- $\beta 1$ and $\alpha$ SMA and subsequently induce liver fibrosis. Furthermore, TGF- $\beta 1$ neutralization in vitro revealed that TGF- $\beta 1$ was important for the fibrotic effect of rLIGHT, which was consistent with studies that showed a central role of TGF- $\beta$ in directly promoting fibrosis ${ }^{41,42}$. These findings expand our understanding of the complex regulatory networks among LIGHT, LT $\beta R$, JNK, and TGF- $\beta 1$.

Although there was a promotional effect on liver fibrosis in vitro, the role of LIGHT in vivo remains unclear. Herro et al. found that rLIGHT injection upregulated the major profibrotic factors TGF- $\beta$, IL-13, and TSLP and had major roles in pulmonary and skin fibrosis. LIGHT-deficient mice also exhibit defective fibrotic activity in the skin ${ }^{16,18,19}$. Because splenectomy improved liver fibrosis induced by ConA, we examined whether splenectomy had the same effect after the injection of rLIGHT. If rLIGHT had a direct profibrotic role in liver fibrosis, splenectomy would not improve liver fibrosis after the injection of rLIGHT. The results showed that the injection of rLIGHT into mice exacerbated fibrosis in liver tissue and abolished the effect of splenectomy. Furthermore, blockade of LIGHT in animal models of liver fibrosis without splenectomy significantly improved liver fibrosis. These results illustrated the direct profibrotic role of LIGHT in liver fibrosis.

Our results suggest that LIGHT promotes liver fibrosis by binding to LT $\beta R$ to activate the phosphorylation of JNK. As a result, the secretion of TGF- $\beta 1$ by macrophages increases, resulting in liver fibrosis. Furthermore, serum LIGHT levels were reduced after splenectomy and indirectly improved fibrosis in liver tissue.

\footnotetext{
Acknowledgements

The study was funded by the National Natural Science Foundation of China (81871258 and 81700533), the Key R \& D Program in Shaanxi Province (2017SF_116), and the Innovation and Development Foundation of the Second Hospital Affiliated to Air Force Military Medical University (2017LCYJ003, 2018JSYJ010, and 2019LCYJ005). We thank Mitchell Arico from Liwen Bianji, Edanz Group China (www.liwenbianji.cn/ac) for editing the English text of a draft of this manuscript.
} 


\section{Author details}

${ }^{1}$ Department of General Surgery, The Second Affiliated Hospital of Air Force Military Medical University, 710038 Xi'an, Shaanxi, China. ${ }^{2}$ Department of Emergency, The First Affiliated Hospital of Air Force Military Medical University, 710032 Xi'an, Shaanxi, China. ${ }^{3}$ Department of Immunology, School of Basic Medical Sciences, Air Force Military Medical University, 710032 Xi'an, Shaanxi, China. ${ }^{4}$ Transplant Immunology Laboratory, School of Basic Medical Sciences, Air Force Military Medical University, 710032 Xi'an, Shaanxi, China. ${ }^{5}$ Institute of Medical Research, Northwest Polytechnic University, 710072 Xi'an, Shaanxi, China

\section{Conflict of interest}

The authors declare no competing interests.

\section{Publisher's note}

Springer Nature remains neutral with regard to jurisdictional claims in published maps and institutional affiliations.

Supplementary information The online version contains supplementary material available at https://doi.org/10.1038/s12276-021-00574-2.

Received: 2 September 2020 Revised: 15 January 2021 Accepted: 18 January 2021.

Published online: 3 March 2021

\section{References}

1. Lin, L. et al. Nanodrug with $\mathrm{ROS}$ and $\mathrm{pH}$ dual-sensitivity ameliorates liver fibrosis via multicellular regulation. Adv. Sci. 7, 1903138 (2020).

2. Yada, A. et al. Splenectomy attenuates murine liver fibrosis with hypersplenism stimulating hepatic accumulation of Ly-6C(lo) macrophages. J. Hepatol. 63, 905-916 (2015).

3. Li, L. et al. The spleen in liver cirrhosis: revisiting an old enemy with novel targets. J. Transl. Med. 15, 111 (2017).

4. Wang, P. et al. Spleen regulates hematopoietic stem/progenitor cell functions through regulation of EGF in cirrhotic hypersplenism. Dig. Dis. Sci. 63, 1860-1867 (2018).

5. Huang, N. et al. Spleen-Associated Effects on Immunity in Hepatitis B VirusRelated Cirrhosis with Portal Hypertension. J. Interferon Cytokine Res. 39, 95-105 (2019).

6. Aoyama, T. et al. Spleen-derived lipocalin-2 in the portal vein regulates Kupffer cells activation and attenuates the development of liver fibrosis in mice. Lab. Invest. 97, 890-902 (2017).

7. Akahoshi, T. et al. Role of the spleen in liver fibrosis in rats may be mediated by transforming growth factor beta-1. J. Gastroenterol. Hepatol. 17, 59-65 (2002).

8. Nomura, Y. et al. Influence of splenectomy in patients with liver cirrhosis and hypersplenism. Hepatol. Res. 44, E100-E109 (2014).

9. Tomikawa, M. et al. Effectiveness of gastric devascularization and splenectomy for patients with gastric varices. J. Am. Coll. Surg. 191, 498-503 (2000).

10. Zhan, X. L., Ji, Y. \& Wang, Y. D. Laparoscopic splenectomy for hypersplenism secondary to liver cirrhosis and portal hypertension. World J. Gastroenterol. 20 5794-5800 (2014).

11. Murata, K. et al. Splenectomy improves liver function in patients with liver cirrhosis. Hepatogastroenterology 55, 1407-1411 (2008).

12. Li, L. et al. The spleen promotes the secretion of CCL2 and supports an M1 dominant phenotype in hepatic macrophages during liver fibrosis. Cell Physiol. Biochem. 51, 557-574 (2018).

13. del Rio, M. L. et al. Immunotherapeutic targeting of LIGHT/LTRR/HVEM pathway fully recapitulates the reduced cytotoxic phenotype of LIGHTdeficient T cells. MAbs 8, 478-490 (2016).

14. Murphy, M. et al. Expression of the lymphotoxin beta receptor on follicular stromal cells in human lymphoid tissues. Cell Death Differ. 5, 497-505 (1998)

15. Herro, R. \& Croft, M. The control of tissue fibrosis by the inflammatory molecule LIGHT (TNF Superfamily member 14). Pharm. Res. 104, 151-155 (2016).
16. Doherty, T. A. et al. The tumor necrosis factor family member LIGHT is a target for asthmatic airway remodeling. Nat. Med. 17, 596-603 (2011).

17. Lee, W. H. et al. Tumor necrosis factor receptor superfamily 14 is involved in atherogenesis by inducing proinflammatory cytokines and matrix metalloproteinases. Arterioscler. Thromb. Vasc. Biol. 21, 2004-2010 (2001).

18. Herro, R., Antunes, R. D. S., Aguilera, A. R., Tamada, K. \& Croft, M. The tumor necrosis factor superfamily molecule LIGHT promotes keratinocyte activity and skin fibrosis. J. Invest. Dermatol. 135, 2109-2118 (2015).

19. Herro, R., Da Silva Antunes, R., Aguilera, A. R., Tamada, K. \& Croft, M. Tumor necrosis factor superfamily 14 (LIGHT) controls thymic stromal lymphopoietin to drive pulmonary fibrosis. J. Allergy Clin. Immunol. 136, 757-768 (2015).

20. Wang, Y. et al. Splenectomy promotes macrophage polarization in a mouse model of concanavalin A- (ConA-) induced liver fibrosis. Biomed. Res. Int. 2019, 5756189 (2019)

21. Wang, J. et al. The regulation of $T$ cell homeostasis and autoimmunity by $T$ cell-derived LIGHT. J. Clin. Investig. 108, 1771-1780 (2001).

22. Xu, M. et al. LECT2, a ligand for Tie1, plays a crucial role in liver fibrogenesis. Cell 178, 1478-92.e20 (2019)

23. Li, J. et al. ADAR1 attenuates allogeneic graft rejection by suppressing miR-21 biogenesis in macrophages and promoting M2 polarization. FASEB J. 32, 5162-5173 (2018)

24. Nielsen, S. R. et al. Macrophage-secreted granulin supports pancreatic cancer metastasis by inducing liver fibrosis. Nat. Cell Biol. 18, 549-560 (2016).

25. Granger, S. W. \& Rickert, S. LIGHT-HVEM signaling and the regulation of T cellmediated immunity. Cytokine Growth Factor Rev. 14, 289-296 (2003).

26. Cai, X. et al. CXCL6-EGFR-induced Kupffer cells secrete TGF- $\beta 1$ promoting hepatic stellate cell activation via the SMAD2/BRD4/C-MYC/EZH2 pathway in liver fibrosis. J. Cell Mol. Med. 22, 5050-5061 (2018).

27. Wynn, T. A. \& Vannella, K. M. Macrophages in tissue repair, regeneration, and fibrosis. Immunity 44, 450-462 (2016).

28. Tang, W. P. et al. Splenectomy enhances the therapeutic effect of adipose tissue-derived mesenchymal stem cell infusion on cirrhosis rats. Liver Int. 36, 1151-1159 (2016)

29. Wang, $K$. et al. TGF- $\beta 1 /$ p65/MAT2A pathway regulates liver fibrogenesis via intracellular SAM. EBioMedicine 42, 458-469 (2019).

30. Wang, M. J. et al. Non-invasive evaluation of liver stiffness after splenectomy in rabbits with CCl(4)-induced liver fibrosis. World J. Gastroenterol. 22, 10166-10179 (2016)

31. Xiang, D. M. et al. The HLF/LL-6/STAT3 feedforward circuit drives hepatic stellate cell activation to promote liver fibrosis. Gut 67, 1704-1715 (2018).

32. Giusto, M. et al. Skeletal muscle myopenia in mice model of bile duct ligation and carbon tetrachloride-induced liver cirrhosis. Physiol. Rep. 5, e13153 (2017)

33. Schneider, K., Potter, K. G. \& Ware, C. F. Lymphotoxin and LIGHT signaling pathways and target genes. Immunol. Rev. 202, 49-66 (2004).

34. Otterdal, $K$. et al. Increased serum levels of LIGHT/TNFSF14 in nonalcoholic fatty liver disease: possible role in hepatic inflammation. Clin. Transl. Gastroenterol. 6, e95 (2015).

35. Wang, J. et al. The critical role of LIGHT in promoting intestinal inflammation and Crohn's disease. J. Immunol. 174, 8173-8182 (2005).

36. Pierer, M. et al. The TNF superfamily member LIGHT contributes to survival and activation of synovial fibroblasts in rheumatoid arthritis. Rheumatology 46 , 1063-1070 (2007).

37. Kotani, H. et al. Increased plasma LIGHT levels in patients with atopic dermatitis. Clin. Exp. Immunol. 168, 318-324 (2012).

38. Seki, E. \& Schwabe, R. F. Hepatic inflammation and fibrosis: functional links and key pathways. Hepatology 61, 1066-1079 (2015).

39. Kou, Y. et al. LIGHT/TNFSF14 signaling attenuates beige fat biogenesis. FASEB J. 33, 1595-1604 (2019).

40. Kim, Y. S., Nedospasov, S. A. \& Liu, Z. G. TRAF2 plays a key, nonredundant role in LIGHT-lymphotoxin beta receptor signaling. Mol. Cell Biol. 25, 2130-2137 (2005).

41. El-Maadawy, W. H., Hammam, O. A., Seif El-Din, S. H. \& El-Lakkany, N. M. aLipoic acid modulates liver fibrosis: a cross talk between TGF- $\beta 1$, autophagy, and apoptosis. Hum. Exp. Toxicol. 39, 440-450 (2020).

42. $\mathrm{Xu}, \mathrm{Y}$. et al. A positive feedback loop of TET3 and TGF- $\beta 1$ promotes liver fibrosis. Cell Rep. 30, 1310-8.e5 (2020). 